\title{
Influencia de Finos de Perlita en Hormigones
}

\section{Influence of perlite fines in concretes}

Presentación: 08/05/2021

Aprobación: 17/05/2021

\section{Verónica Artigas}

Instituto de Investigaciones para la Industria Química - Universidad Nacional de Salta - Argentina veronicaartigas6@gmail.com

\section{María Josefina Positieri}

Facultad Regional Córdoba - Universidad Tecnológica Nacional - Argentina

mpositieri@gmail.com

\section{María Virginia Quintana}

Instituto de Investigaciones para la Industria Química - Universidad Nacional de Salta - Argentina quintanamvirginia@gmail.com

\section{Ángel Oshiro}

Facultad Regional Córdoba - Universidad Tecnológica Nacional - Argentina

oshiroangel@gmail.com

\section{Resumen}

La perlita es una roca de origen volcánico utilizada en múltiples industrias. En San Antonio de los Cobres (Salta-Argentina) se sitúan canteras de extracción de perlita, donde es sometida a un proceso mecánico que genera desechos del mineral llamados "finos de perlita" (FP).

Este trabajo presenta el análisis de hormigones con la incorporación de FPcomo adición mineral al contenido de cemento y como reemplazo del cemento, planteando dosificaciones con el fin de comprobar si la perlita puede ser considerada como una adición activa o simplemente modifica ciertas propiedades del hormigón. Se evaluaron en estado fresco el aspecto, la consistencia y el tiempo de fraguado y en estado endurecido la resistencia a compresión, módulo de elasticidad, absorción y velocidad de pulso ultrasónico.

Los resultados obtenidos con incorporación de FP hasta el 10 por ciento de reemplazo del cemento muestran que es posible la utilización de este material como adición activa.

Palabras claves:Hormigón, Perlita, Adición mineral, Durabilidad

\section{Abstract}

Perlite is a volcanic rockused in multiple industries. In San Antonio de losCobres (SaltaArgentina) there are perlite extraction quarries, the rock is subjected to a mechanical 
process that generate ore waste called "perlite fines" (PF).

This research presents the analysis of concrete with the incorporation of $\mathrm{PF}$ as a mineral addition to the cement content and as a cement replacement, proposing dosages in order to check whether perlite can be considered as an active addition or simply as a material that modifies certain properties of concrete. In the fresh state, appearance, consistency and setting time were evaluated, and in the hardened state, compressive strength, modulus of elasticity, absorption and ultrasonic pulse velocity were measured.

The results obtained with the incorporation of $\mathrm{PF}$ up to 10 percent replacement of cement show that it is possible to use this material as an active addition.

Keywords: Concrete, Perlite, Mineral admixture, Durability

\section{Introducción}

Mundialmente, el hormigón es el material artificial más utilizado, con una producción anual que se estima en los 10.000 millones de toneladas(Bonnet et al., 2019). La industria del hormigón resulta sumamente contaminante para el medio ambiente debido a que uno de sus principales compuestos es el cemento, cuya producción genera alrededor del 7\% de las emisiones mundiales de dióxido de carbono generadas por el hombre(Pal, 2018). Además, la producción de cemento implica un elevado consumo de energía y de materia prima (piedra caliza y arcilla).

Sin duda el consumo de hormigón se va incrementando año a año con los nuevos avances de la industria de la construcción y por eso desde el punto de vista de la ciencia de los materiales es importante el desarrollo de hormigones sustentables que no sólo contemplen mantener y mejorar la calidad de éstos como material estructural sino que se lo considere un elemento sustentable de construcción(Raggioti, 2015).La energía que se emplea en las obras de ingeniería, tanto en la fase de construcción, como en la de operación o uso de éstas, depende en gran medida, del diseño, de la tecnología y de los materiales empleados (Astori et al., 2005).

Así, la posibilidad de utilizar residuos de otras industrias para la elaboración de hormigones se constituye en un objetivo de desarrollo sustentable. Este trabajo evalúa los efectos de la adición de perlita finamente molida en el hormigón, analizando la viabilidad de emplearla como adición o reemplazo de parte del cemento, disminuyendo de esta forma el consumo de clínker y consecuentemente minimizando la emisión de $\mathrm{CO} 2$ y produciendo un ahorro energético.

\section{La perlita}

La perlita es un vidrio volcánico silíceo que expande hasta 20 veces su volumen original cuando se calienta rápidamente, dando origen a la perlita expandida que tiene múltiples aplicaciones en diversos campos. En usos hortícolas, cuando se agrega al suelo, se usa para proporcionar retención de humedad y aireación sin compactación. Las aplicaciones de la perlita expandida en la construcción son numerosas porque es liviana, resistente al fuego y un excelente aislante (U.S. Geological Survey, 2021).

Los principales yacimientos de perlita en América del Sur y en Argentina se encuentran al norte del país, en la provincia de Salta, ubicados en una región denominada "Puna". La Puna argentina es una región eminentemente volcánica y en ella se han encontrado numerosas 
coladas y domos de vidrio que se han perlitizado. Si bien se conocen una decena de localidades en Salta y algunas manifestaciones en Catamarca, los principales distritos ricos en perlita son los del Volcán Quevar, Ramadas y Rupasca. El yacimiento mejor ubicado es el de Ramadas a sólo $15 \mathrm{~km}$ al norte de San Antonio de los Cobres. Al ser rocas, su tipo de explotación recibe el nombre de canteras (Alonso, 2014).

La perlita extraída en las canteras de la zona de San Antonio de los Cobres es sometida a un proceso mecánico con el fin de llevar la roca a un tamaño más pequeño y así transportarla a la planta procesadora para su expansión. Durante las operaciones de molienda del material mediante chancadora y molino de barras se genera una fracción fina que se considera un desecho, es acumulada al aire libre formándose grandes depósitos. Es un polvo sumamente fino, depositado en una zona ventosa que produce la dispersión de las partículas originando inconvenientes para los habitantes y la fauna de la zona (Artigas et al., 2016).

Aproximadamente el $20 \%$ de la perlita extraída se convierte en desecho. Considerando que en una de las canteras de la zona se extraen diariamente cerca de 96 toneladas, se producen unas 19 toneladas de finos de perlita por día. En la Figura 1 se pueden observar depósitos de los desechos de perlita acumulados al aire libre.

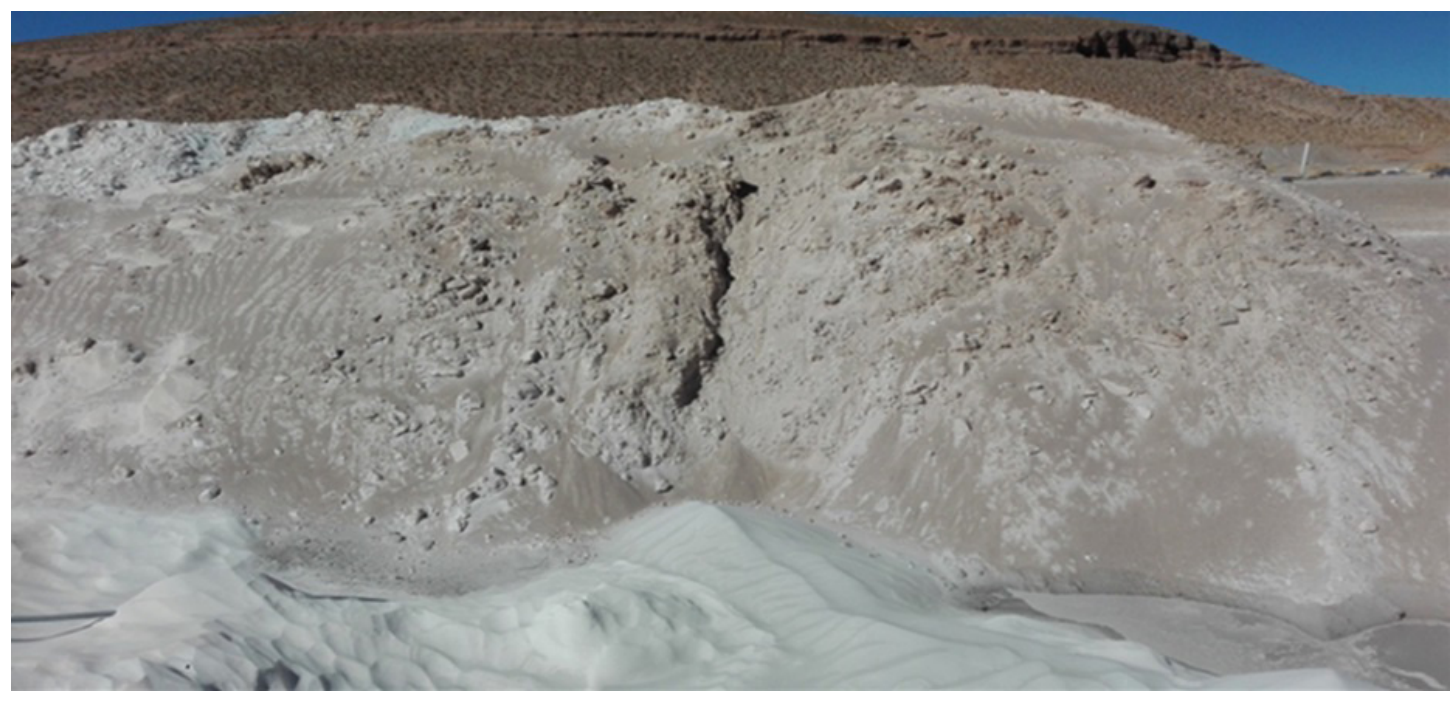

Fig. 1: Desechos de finos de perlita acumulados al aire libre. Foto propia

\section{Caracterización de finos de perlita cruda}

- Densidad: 2.64, obtenida por el método del picnómetro bajo el lineamiento de la norma IRAM 12510.

- Superficie específica BET: $5.28 \mathrm{~m}^{2} / \mathrm{gr}$, obtenida mediante un sortómetro BET, el cual permite determinar la superficie específica general de sólidos dispersos o sólidos porosos midiendo la cantidad de gas adsorbido físicamente de acuerdo con el método Brunauer, Emmett y Teller.

- Difracción láser: se presenta en la Figura 2 la distribución granulométrica obtenida de una muestra del material.El 90 por ciento de las partículas de los FP tiene un tamaño inferior a $75 \mu \mathrm{m}$. 


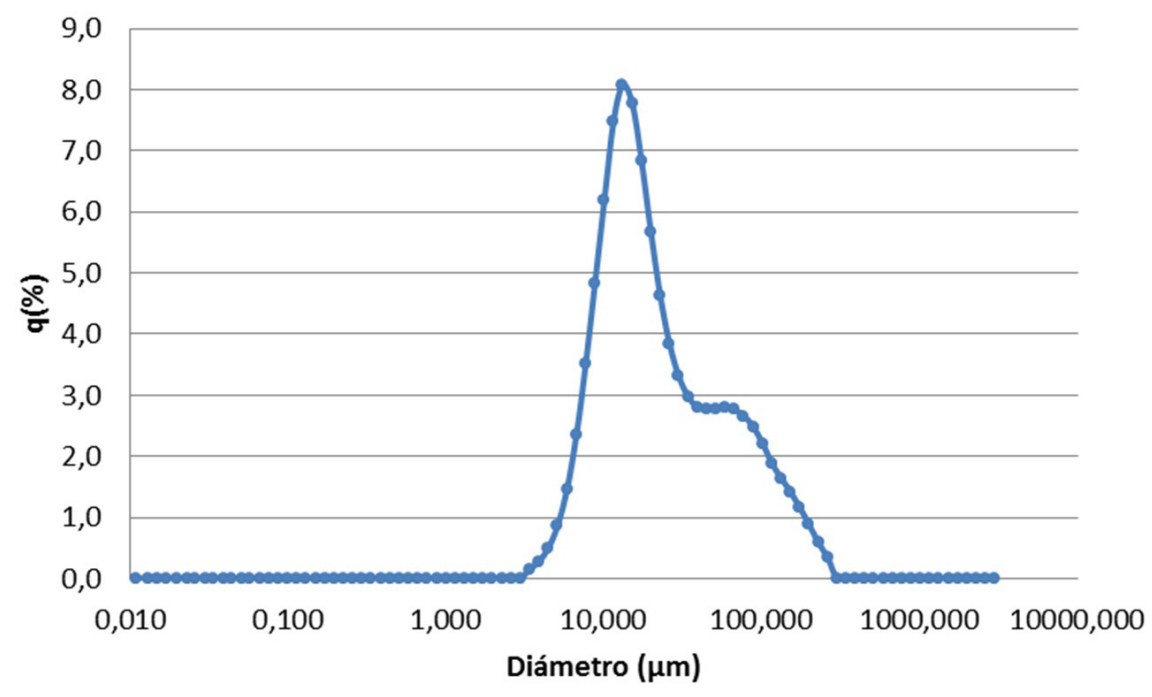

Fig. 2: Distribución granulométrica de los FP

- Microscopía electrónica de barrido (SEM): En la Figura 3 se presenta una de las imágenes SEM de las partículas de FP, donde se puede apreciar que los lados de la misma consisten en caras planas.

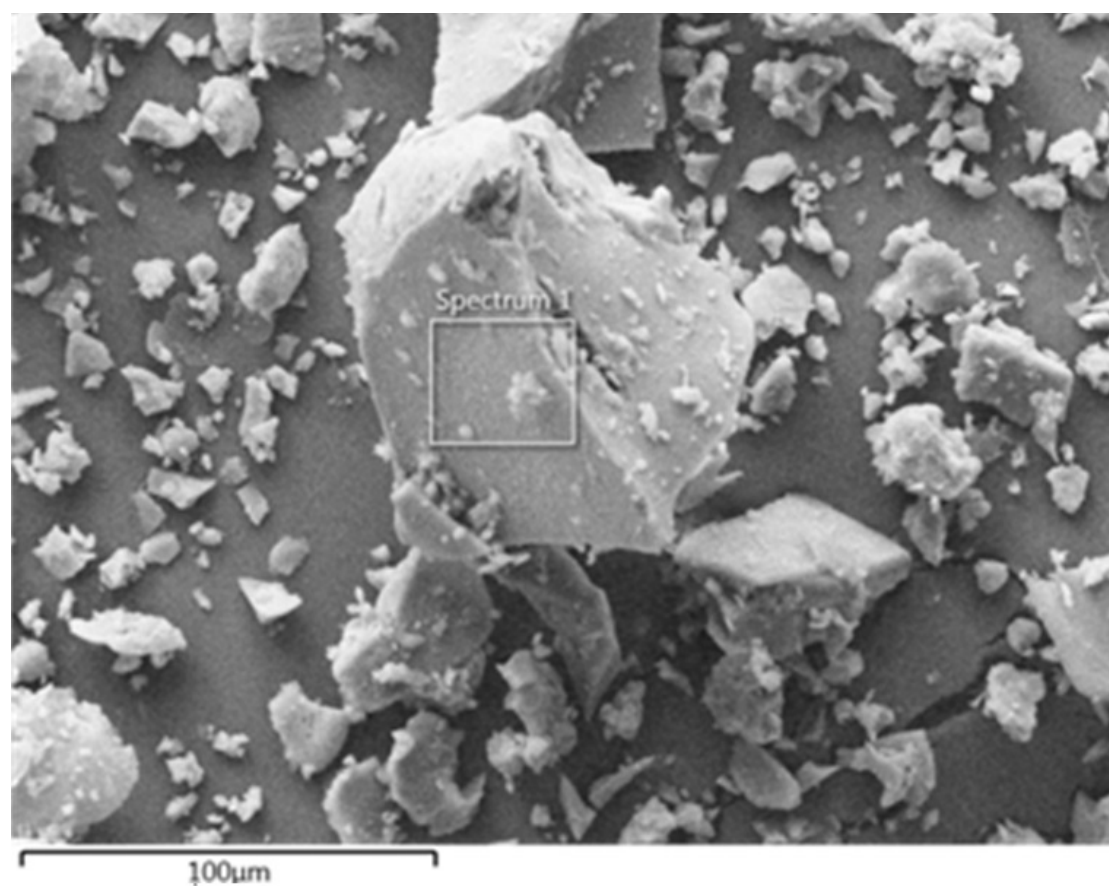

Fig. 3: Imagen de los FP obtenida por microscopía electrónica de barrido (SEM) 
- Difractometría de rayos X (DRX): El difractogrma de la muestra de FP (Fig. 4) presenta los picos característicos de minerales de sílice (como el cuarzo) entre $20-22^{\circ}$ y $26-28^{\circ}$ y una forma curva en la región entre 40 y $90^{\circ}$ lo que indica una ausencia de estructura mineral definida.

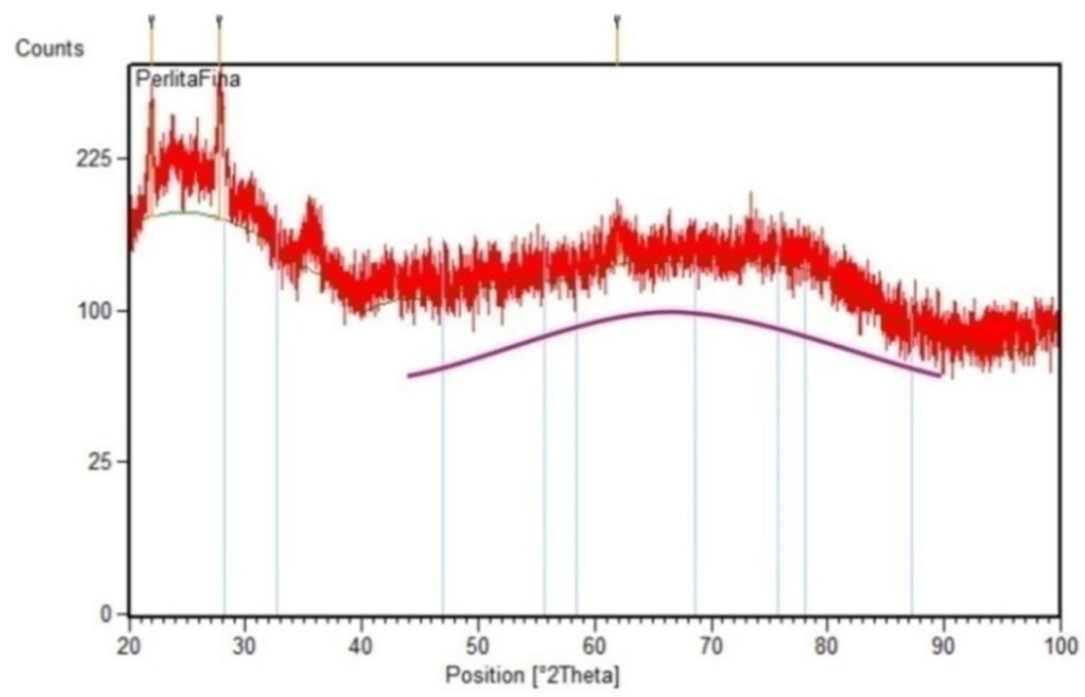

Fig. 4: Patrón de difracción de los FP

- Análisis químico: En la Tabla 1 se presenta la composición química de los FP. Se observa un elevado contenido de silicio y aluminio. Además, el contenido de H2O se encuentra por encima del $2 \%$, límite inferior a partir del cual el material presenta una fácil y rápida expansión al ser expuesto a altas temperaturas.

\begin{tabular}{|c|c|c|c|c|c|c|c|c|c|c|}
\hline Compuesto & Si & Al & Fe & Ca & Mg & Na & K & Sulfato & $\mathbf{O}_{2}$ & $\mathbf{H}_{2} \mathbf{O}$ \\
\hline$\%$ & $30-36$ & $6-8$ & $0-1$ & $0-1$ & $0-1$ & $2.5-5$ & $2-4$ & exenta & $44.5-47.5$ & $3-5$ \\
\hline
\end{tabular}

Tabla 1: Composición química de los FP

- Capacidad puzolánica: De acuerdo al método de Frattini, norma IRAM 1651. Este es un método químico que determina la cantidad máxima de hidróxido de calcio con la que la puzolana se puede combinar y a la velocidad con la cual ocurre esta reacción. La misma depende de la naturaleza y proporción de las fases activas presentes en la puzolana, de la relación cal-puzolana de la mezcla, de la finura (o superficie específica) de la puzolana y de la temperatura de reacción.

El coeficiente de puzolanizidad a la edad de 8 días es de 1,17 y a los 15 días de 1,05, lo cual no indicaría actividad puzolánica de los FP desde el punto de vista químico. Sin embargo, estos resultados no son determinantes, es necesaria la realización de ensayos mecánicos sobre morteros u hormigones para evaluar el posible efecto puzolánico de los FP y su 
evolución en el tiempo.

\section{Desarrollo}

Para verificar la influencia de la utilización de FP se planteó la elaboración de hormigones con adición y reemplazo de parte del cemento por FP, partiendo de un hormigón patrón (HP0). Se definió la adición de perlita finamente molida en porcentajes del 5, 10 y 15\% del peso de cemento conformando la serie $\mathrm{HP}+\mathrm{y}$ la serie con reemplazo del cemento por perlita en los mismos porcentajes mencionados anteriormente se denominó HP-.

Los materiales empleados fueron:

- Cemento portland fillerizado: CPF 40 (densidad 3.09)

- Finos de perlita: partículas menores a 0.075 mm, densidad 2.64; la Tabla 1 muestra su composición química

- Agregado fino: arena silícea natural (densidad 2.65, módulo de finura 2.23)

- Agregado grueso: canto rodado (densidad 2.65, módulo de finura 5.97, TMAG 19 mm)

- Aditivo superfluidificante a base de policarboxilatos (residuo sólido 35\%).

La dosificación del hormigón patrón (HP0) tiene las siguientes características:

- $450 \mathrm{~kg} / \mathrm{m}^{3}$ de cemento

- Relación agua/cemento de 0,42

- Volumen de agregados: $42 \%$ de arena y $52 \%$ de agregado grueso

- Aditivo Superfluidificante necesario para alcanzar un asentamiento de $14 \pm 1 \mathrm{~cm}$

La serie con la adición de perlita finamente molida en porcentajes de 5, 10 y 15\% del peso de cemento se identifica como serie HP+. La serie HP- corresponde al reemplazo del cemento por perlita en los mismos porcentajes mencionados anteriormente.

En la Tabla 2 se presentan las dosificaciones consideradas, detallando el contenido por metro cúbico de cada material constituyente.El valor numérico colocado a la derecha del signo (+ ó -) indica el porcentaje de perlita que contiene la mezcla, en relación al peso del cemento.

\begin{tabular}{|c|c|c|c|c|c|c|c|c|}
\hline Materiales & Unidades & HP0 & HP+5 & HP+10 & HP+15 & HP-5 & HP-10 & HP-15 \\
\hline Cemento & {$\left[\mathrm{kg} / \mathrm{m}^{3}\right]$} & 450 & 450 & 450 & 450 & 428 & 405 & 383 \\
\hline Agua & {$\left[\mathrm{kg} / \mathrm{m}^{3}\right]$} & 190 & 190 & 190 & 190 & 190 & 190 & 190 \\
\hline Finos de Perlita & {$\left[\mathrm{kg} / \mathrm{m}^{3}\right]$} & 0 & 22 & 45 & 67 & 22 & 45 & 67 \\
\hline Agregado Fino & {$\left[\mathrm{kg} / \mathrm{m}^{3}\right]$} & 738 & 729 & 719 & 709 & 737 & 735 & 734 \\
\hline Agregado Grueso & {$\left[\mathrm{kg} / \mathrm{m}^{3}\right]$} & 1019 & 1006 & 993 & 979 & 1017 & 1015 & 1013 \\
\hline $\begin{array}{c}\text { Aditivo } \\
\text { Superfluidificante }\end{array}$ & {$[\%]$} & 0.22 & 0.22 & 0.22 & 0.22 & 0.22 & 0.22 & 0.22 \\
\hline
\end{tabular}

Tabla 2: Dosificaciones para un metro cúbico de hormigón 
Para obtener un asentamiento de $14 \pm 1 \mathrm{~cm}$ en el hormigón patrón fue necesaria una dosis de aditivo superfluidificante igual al $0.22 \%$ con respecto al peso del material cementante, dosis que se mantuvo constante entodos los hormigones.

Cabe destacar que los finos de perlita y el cemento fueron homogeneizados previamente a ser incluidos en la mezcla y que el aditivo superfluidificante se incorporó diluido en el agua de mezclado correspondiente.

\section{Métodos de ensayo}

Método de ensayo de la consistencia por el cono de Abrams (Norma IRAM 1536)

La importancia de conocer la consistencia del hormigón radica en el hecho de que está directamente ligada a la trabajabilidad del mismo. El American Concrete Institute (ACI 116R00) describe la trabajabilidad como "la propiedad del hormigón fresco que determina la facilidad con que puede ser mezclado, colocado, consolidado y terminado en una condición homogénea".

\section{Método de determinación de la densidad, el rendimiento y el contenido de aire (Norma IRAM 1562)}

La densidad es un valor que que se determina en pruebas preliminares, cuando se utiliza por primera vez la mezcla en obra o cuando se modifique, como en este caso, alguna de las materias primas o la dosificación del hormigón.

\section{Determinación del tiempo de fraguado. Método de resistencia a la penetración (Norma IRAM 1662)}

La determinación del tiempo de fraguado es importante cuando se utilizan adiciones minerales ya que es un período en el cual las reacciones químicas entre el cemento y el agua conducen a un proceso que, mediante diferentes velocidades de reacción, generan calor y dan origen a nuevos compuestos;estos compuestos en la pasta de cemento producen el endurecimiento y aglutinan al agregado de la mezcla de hormigón, adquiriendo cierta resistencia.

El fraguado inicial define el límite de manejo, o sea el tiempo durante el cual el hormigón fresco puede ser mezclado adecuadamente, colocado y compactado; indica el momento en el que la masa ha adquirido rigidez y no puede ser vibrada sin dañar su estructura interna. El fraguado final ocurre entre cuatro y ocho horas después del hormigonado.

\section{Método de ensayo de resistencia a compresión (Norma IRAM 1536)}

El ensayo de compresión constituye el método preferencial para valorar las características del hormigón y su nivel de resistencia en particular. Salvo contadas excepciones, ha sido probado que las propiedades más importantes se relacionan directamente con la resistencia a compresión; además el hormigón no sólo se utiliza preponderadamente para sobrellevar esfuerzos de compresión sino que los códigos y reglamentos se basan fundamentalmente en el conocimiento de esta propiedad.

\section{Métodos de ensayo para la determinación del módulo de elasticidad estático (IRAM 1865)}

El módulo de elasticidad es una medida de la rigidez, o sea de la resistencia del hormigón a la deformación. El hormigón no es un material verdaderamente elástico, pero cuando 
ha endurecido por completo y se ha cargado en forma moderada tiene una curva de esfuerzo de compresión/ deformación que es una recta dentro del rango de los esfuerzos usuales de trabajo.

\section{Método para la determinación de la velocidad de pulsos ultrasónicos (Norma IRAM 1683)}

La velocidad de un pulso ultrasónico depende de las propiedades del hormigón que determinan su rigidez elástica y su resistencia mecánica.La calidad del hormigón una vez endurecido responde a un concepto integrado por numerosas propiedades del material; así es posible conocer los cambios en el hormigón debidos a diferentes causas como por ejemplo comparar la velocidad de onda ultrasónica de un hormigón de referencia y los hormigones en los que se emplean adiciones minerales y correlacionar los resultados obtenidos con la resistencia mecánica.

\section{Absorción de agua}

La absorción de agua de los compuestos cementicios es un buen indicador de su durabilidad en general, ya que la mayoría de los agentes agresivos penetran en el material compuesto utilizando el agua como vía. Cuanto más fácil puede penetrar el agua, mayor es su susceptibilidad a diversas amenazas de durabilidad.

\section{Resultados y análisis}

\section{Asentamiento}

El Reglamento Argentino de Estructuras de Hormigón (CIRSOC 201-2005) establece que el ensayo de asentamiento del cono de Abrams es aplicable para consistencias seca, plástica, muy plástica y fluida.

En los hormigones HP0, HP+5, HP+15, HP-5, HP-10 y HP-15 el tipo de asentamiento fue "normal", es decir que la mezcla en estado fresco se deformó plásticamente sin grandes cambios de forma, siendo este comportamiento el adecuado para evaluar la consistencia por el método de Abrams. En la Fig. 5 se presentan los resultados del ensayo.

Considerando el uso de la perlita como adición en estos hormigones se produce una disminución del asentamiento con respecto al patrón; esto indicaría que la perlita absorbe una parte del agua de mezclado. En el hormigón HP+5, al incluir solamente un 5\% de perlita, la disminución del asentamiento es del $48 \%$ respecto del patrón, mientras que al incluir un $10 \%$ y un $15 \%$ de perlita, el asentamiento se reduce en un promedio del $83 \%$ respecto a la mezcla sin contenido de perlita. Sin embargo esta reducción no afecta la plasticidad de la mezcla, manteniendo su cohesión y sin segregación.

Cuando se considera el uso de la perlita como reemplazo por cemento, el asentamiento disminuye en un 41, 63 y 85\% respectivamente para porcentajes de 5, 10 y 15\%, confirmando que en este caso también la inclusión de perlita finamente molida en la mezcla conlleva una pérdida de consistencia en la misma, presentando una buena cohesión y sin segregación. 


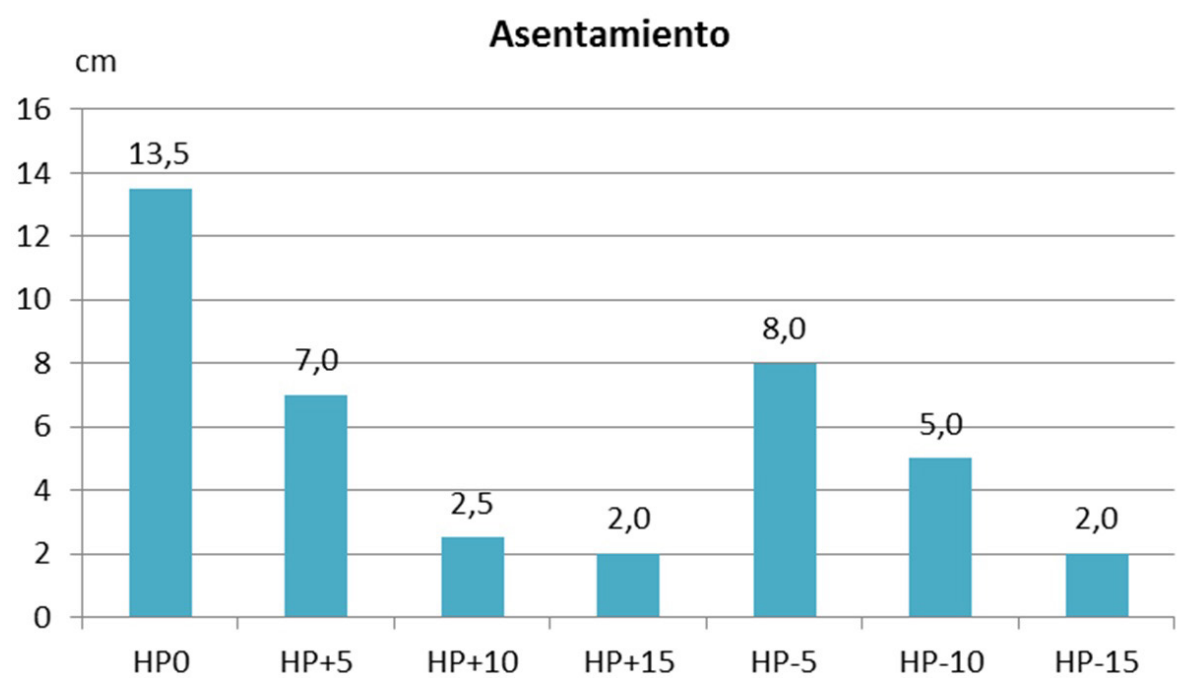

Fig. 5: Asentamiento

Los resultados obtenidos muestran que la utilización de perlita en la mezcla produce una marcada disminución del asentamiento, lo cual indica una disminución en la fluidez. Considerando que para todas las mezclas se utilizó igual porcentaje de aditivo superfluidificante y se mantuvo constante la relación agua/material cementante, la variabilidad en los resultados de asentamiento son exclusivamente causados por el empleo de la perlita y su velocidad de absorción de agua.

\section{Densidad del hormigón}

La densidad de los hormigones con adición o reemplazo de finos de perlita disminuyen con respecto al hormigón de referencia. Sin embargo esta disminución, como máximo del $4 \%$, no es significativa desde el punto de vista práctico.

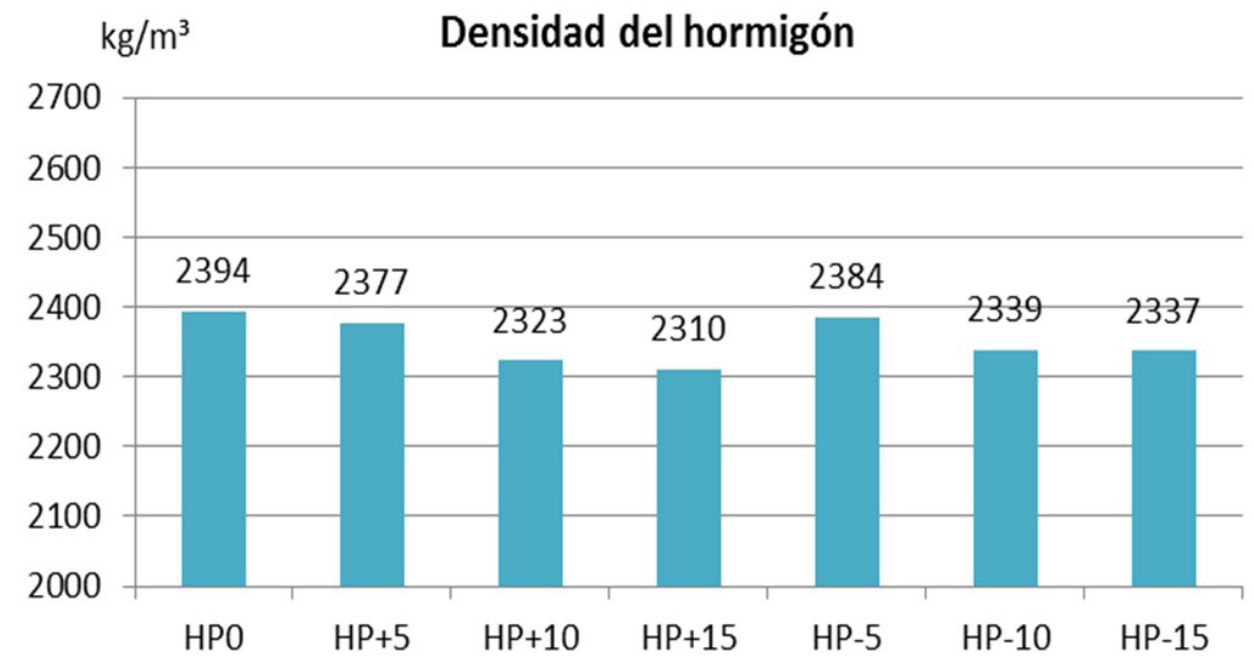

Fig.6: Densidad en estado fresco 


\section{Tiempos de fraguado}

En la Figura 7 se presentan los resultados de los tiempos de inicio y fin de fraguado obtenidos para cada hormigón.El hormigón patrón inicia su fraguado a las 3 horas y 19 minutos y lo culmina a las 4 horas y 15 minutos.

Al adicionar perlita a la mezcla, los tiempos de inicio de fraguado se acortan con respecto al patrón pero no de forma significativa. Por ejemplo, el inicio de fraguado se acelera en un $7 \%$ cuando la adición es del 5\%, en un $14 \%$ cuando se adiciona $10 \%$ de perlita y en un $38 \%$ cuando se incorpora el 15\%. En cuanto a los tiempos de fin de fraguado, la diferencia con el patrón es como máximo un $12 \%$ en el $\mathrm{HP}+15$.

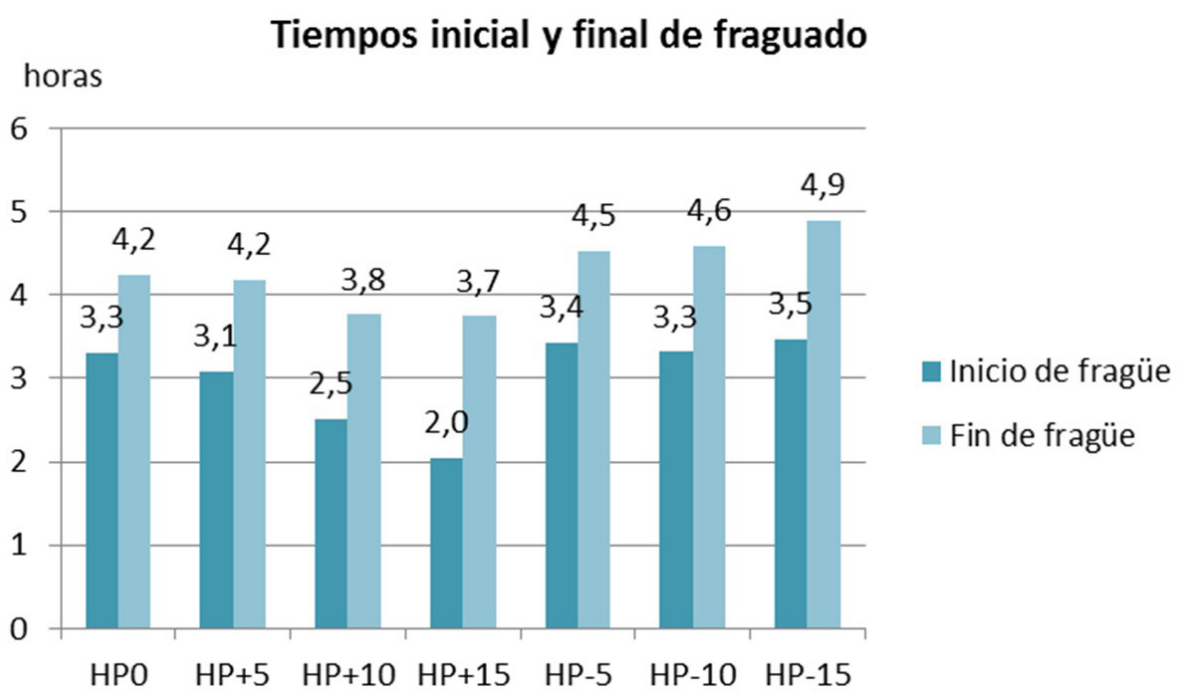

Fig. 7: Tiempos de fraguado inicial y final

En la Fig. 8 se presentan las curvas de resistencia a la penetración en función del tiempo de fraguado. Al reemplazar parte del cemento por perlita en la mezcla, los tiempos de inicio de fraguado prácticamente similares al del hormigón patrón, mientras que para el fin de fraguado son levemente superiores (hasta un 15\% para el caso del HP-15).

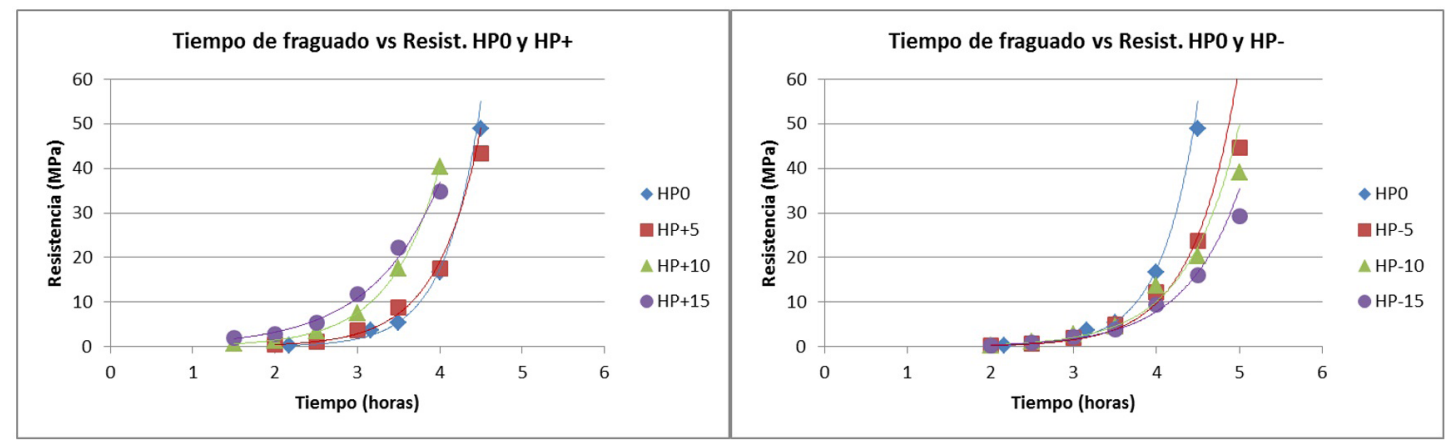

Fig. 8: Tiempos de fraguado 


\section{Resistencia a compresión}

La Figura 9 presenta los resultados de las resistencias a 7, 28 y 90 días obtenidos para cada hormigón. A edades tempranas (7 días) el hormigón patrón es el que presenta la mayor resistencia, seguido por los hormigones HP+5 y HP-5. A medida que se incrementa la cantidad de perlita en la mezcla, las resistencias disminuyen tanto en la serie HP+ (disminución del 12\% en el HP+15) como en la HP- (disminución del 26\% en el HP-15).

A la edad de 28 días, la resistencia a compresión se incrementa en todos los casos con respecto a los 7 días. Las resistencias de los hormigones con mayor contenido de perlita fueron los que sufrieron un mayor aumento; en los hormigones HP+15 y HP-15 el incremento fue de más del 20\%, mientras que en el hormigón patrón fue solamente del 7\%. Las series HP+5, HP +10 y HP-5 superaron los valores de resistencia del HP0, mientras que las de HP+15 y HP-10 fueron apenas inferiores; HP-15 presentó un 13\% menos de resistencia a compresión que HP0.

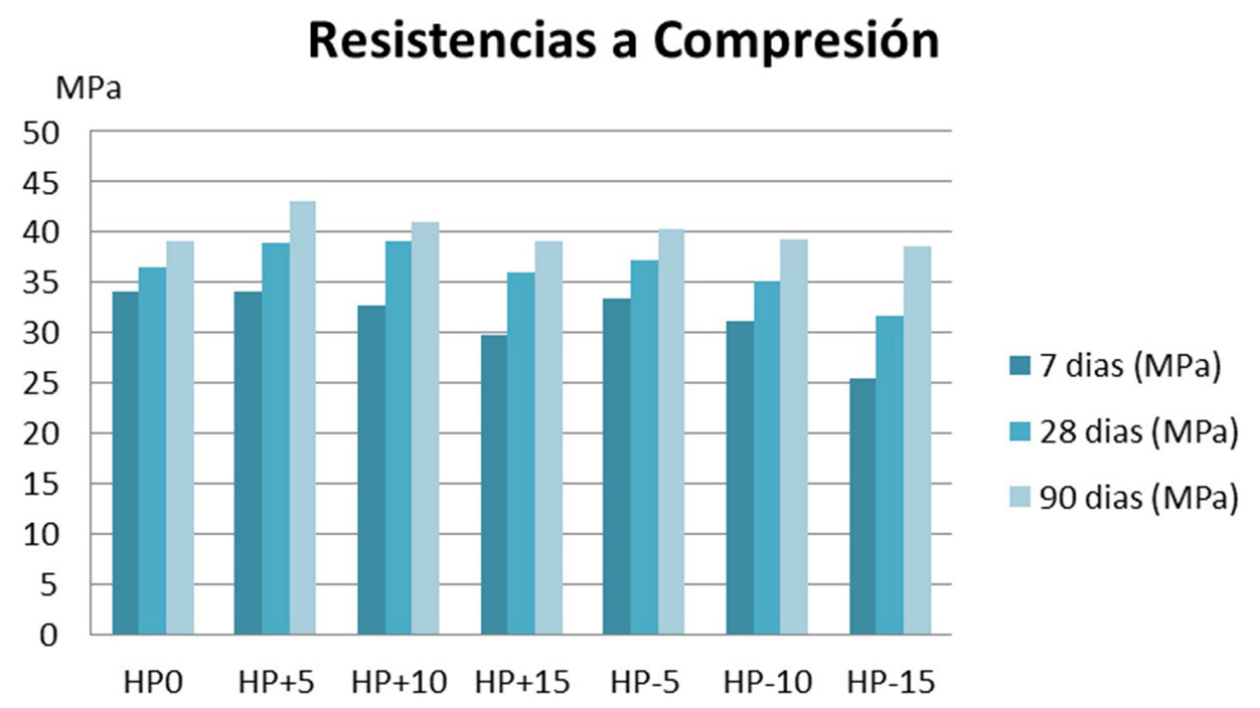

Fig. 9: Resistencias a compresión

Los ensayos a compresión realizados a los 90 días brindan resultados muy interesantes, ya que la evolución de la resistencia en comparación con la obtenida a los 28 días fue superior en los hormigones con perlita (a excepción del hormigón HP+10). El hormigón que alcanza los valores de resistencia más elevados es el HP+5 (un 10\% por encima del patrón), el HP+10 resultó ser un $5 \%$ más resistente que el patrón mientras que el HP+15 alcanzó una resistencia del mismo orden que el hormigón sin contenido de finos de perlita.

Los valores obtenidos para la serie HP+ muestran que la adición de perlita tiene un efecto beneficioso en la resistencia a compresión a medida que aumenta la edad del hormigón. Este efecto disminuye cuando aumenta la cantidad de perlita incorporada, alcanzando un máximo para una relación $\mathrm{P} / \mathrm{C}$ (perlita sobre cemento) en peso de 0.05 e igualando al patrón para una relación $\mathrm{P} / \mathrm{C}$ de 0.15 .

Para la serie HP- los incrementos de las resistencias desde los 28 días hasta los 90 fueron los más significativos, destacándose el hormigón HP-15 el cual aumentó su resistencia en un 
$22 \%$. Un mayor contenido de perlita como reemplazo del cemento muestra un mayor incremento de la resistencia a compresión a largo plazo. Todos los hormigones de esta serie alcanzaron valores de resistencias similares a los del hormigón patrón, aunque cabe mencionar que el más resistente fue el HP-5 (3\% por encima del patrón). Cuando la incorporación de perlita es mayor, la resistencia del hormigón disminuye pero de manera prácticamente insignificante, manifestándose el efecto puzolánico de la perlita.

\section{Módulo de elasticidad}

El módulo de elasticidad es un parámetro muy importante en el análisis de las estructuras de hormigón ya que se emplea en el cálculo de la rigidez de los elementos estructurales. Se ha verificado que el módulo de elasticidad del hormigón (E) crece a medida que crece la resistencia a compresión (f'c). La Tabla 3 presenta los resultados de los módulos de elasticidad obtenidos para cada hormigón en los que se muestra la influencia del empleo de los finos de perlita en las dosificaciones.

\begin{tabular}{|c|c|}
\hline Hormigón & E [GPa] \\
\hline HPO & 23.0 \\
\hline HP+5 & 22.4 \\
\hline HP+10 & 18.5 \\
\hline HP+15 & 19.1 \\
\hline HP-5 & 21.4 \\
\hline HP-10 & 19.7 \\
\hline HP-15 & 17.4 \\
\hline
\end{tabular}

Tabla 3: Módulos de elasticidad

Los códigos y reglamentos proponen diversas relaciones entre $\mathrm{E}$ y f'c como forma de estimación de la rigidez. En la Ecuación 1 se presenta la propuesta del reglamento CIRSOC 2012005 para la estimación del módulo de elasticidad en función de la resistencia a compresión para hormigón de densidad normal.

$$
E c=4700 \sqrt{\left(f_{c}^{\prime}\right)}
$$

Con los resultados obtenidos es posible aplicar la fórmula de la Ecuación 1 con los valores de los promedios de las resistencias a 28 días obtenidas para cada hormigón y verificar que se cumple el concepto de que a mayor resistencia mayor módulo de elasticidad en prácticamente todos los casos. 


\section{Modulo de elasticidad}

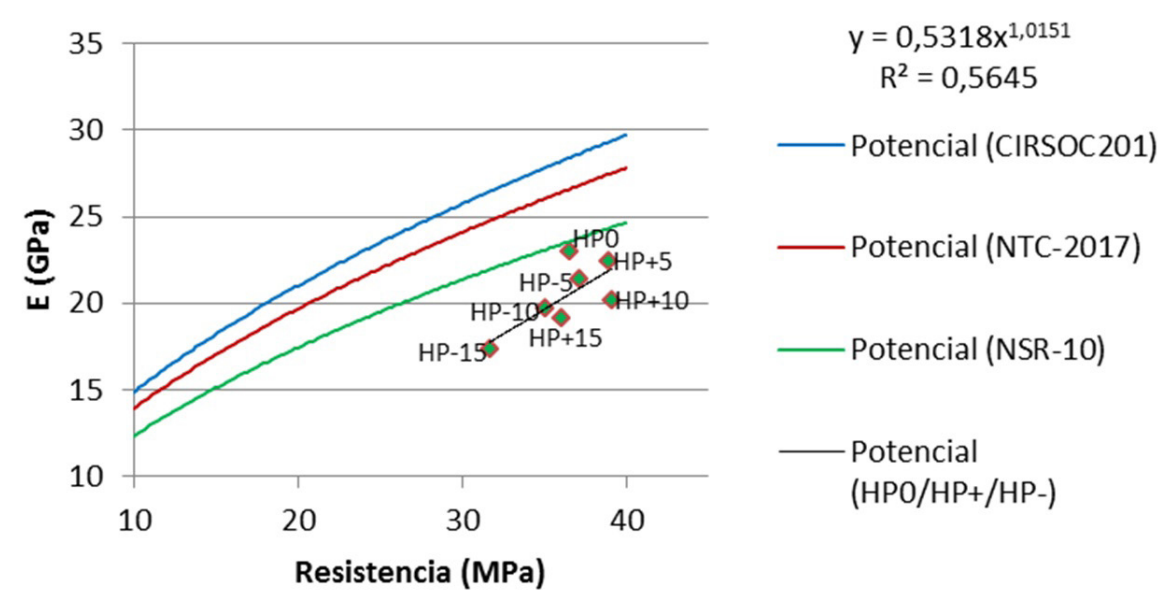

Fig. 10:Curvas de correlación para módulos de elasticidad según distintos Reglamentos

En la Figura 10 se presentangráficamente los valores de $\mathrm{E}$ obtenidos para el grupo de hormigones elaborados junto con las expresionesde módulo de elasticidad para distintos reglamentos internacionales actuales, elReglamento CIRSOC 201-2005 de Argentina, las Normas Técnicas Complementarias (NTC-2017) de México y la Norma Sismo Resistente (NSR-10) de Colombia.

Cualquiera sea la expresión que se use, no se debe perder de vista que el valor que se obtenga es útil solamente a nivel de anteproyecto ya que para el proyecto final de una obra se debe emplear el módulo de elasticidad del hormigón que realmente se usará en la obra.

\section{Velocidad de pulsos ultrasónicos}

En la Figura 11 se presenta la velocidad de onda ultrasónica obtenida en los hormigones por transmisión directa. El hormigón patrón presenta el resultado más alto. La serie con adición de finos de perlita muestra una disminución de la velocidad a medida que aumenta el porcentaje de adición. La serie con reemplazo de finos de perlita muestra un comportamiento similar. 


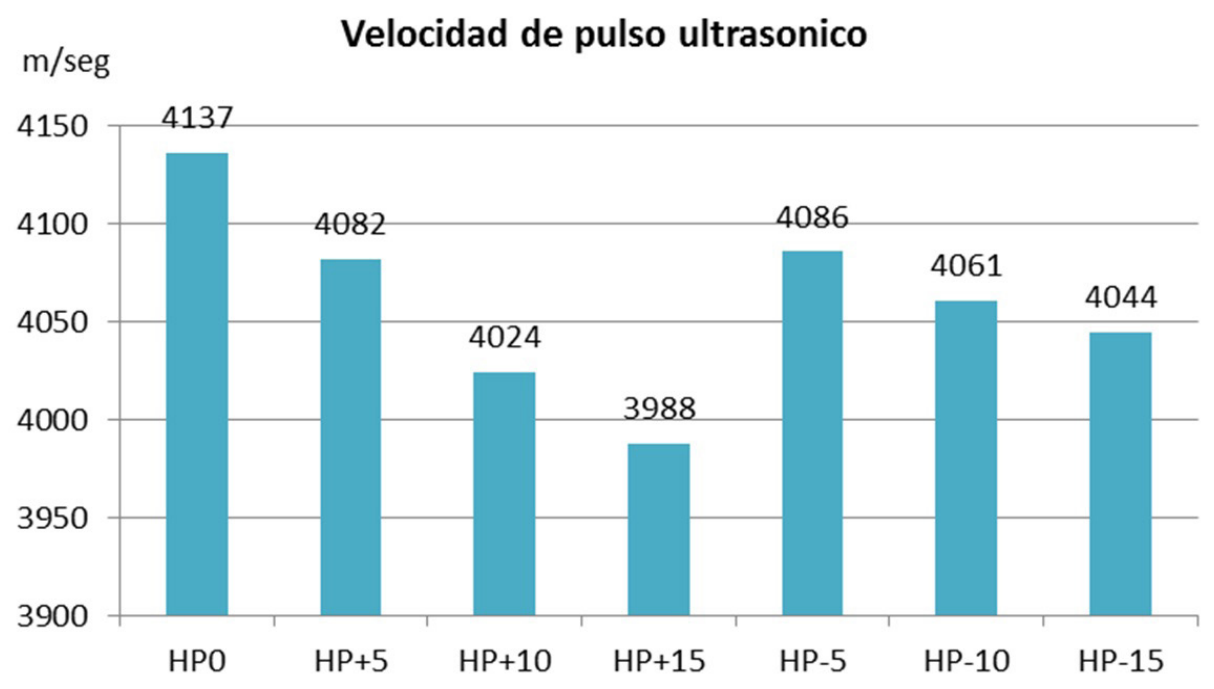

Fig. 11: Velocidad de pulsos ultrasónicos

\section{Absorción de agua}

En la Figura 12 se presentan los resultados de la absorción de agua. El hormigón patrón HP0 muestra el menor valor y los hormigones con finos de perlita aumentan levemente su porcentaje de absorción. Sin embargo no consideran cambios significativos para el desempeño de los hormigones ya que las variaciones no alcanzan al $1 \%$.

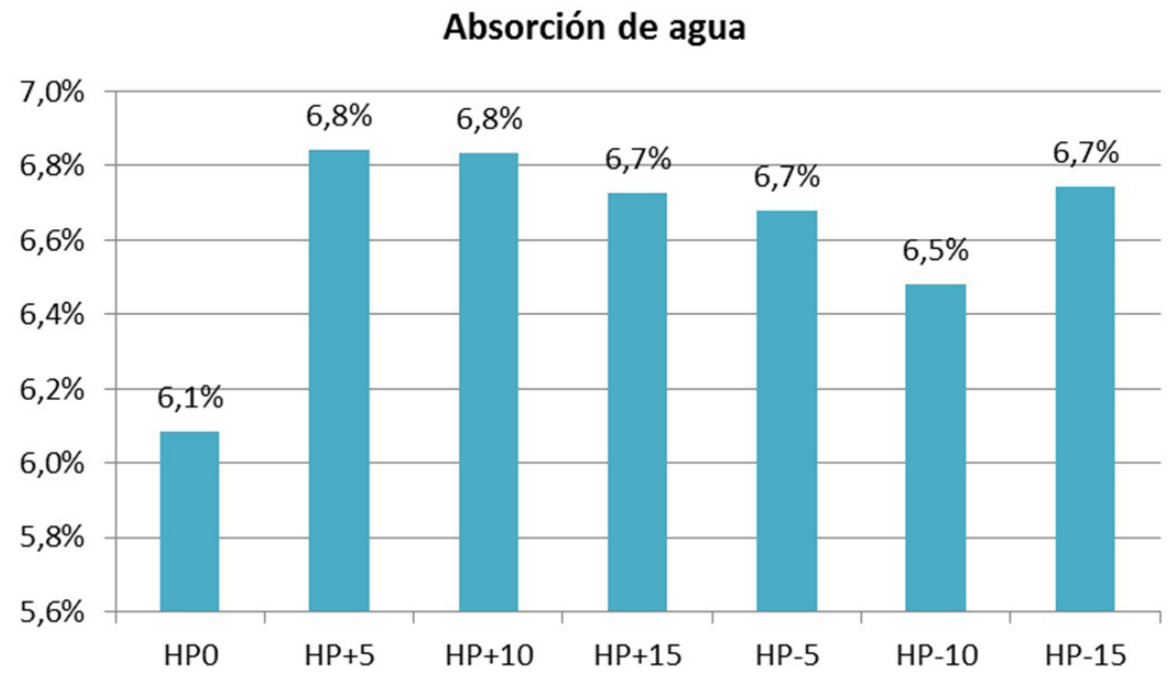

Fig. 12: Absorción de agua 
La absorción de agua puede disminuir por la influencia de la incorporación de materiales muy finos y atribuirse al relleno de poros y a la mejora de la zona de transición interfacial entre la matriz de agregados y cemento. También puede explicarse por la producción significativa de hidratos de silicato de calcio adicionales que dan como resultado el refinamiento de la microestructura y la correspondiente reducción de la absorción.

\section{Conclusiones}

De los resultados obtenidos en este trabajo se puede concluir que la incorporación de finos de perlita en la elaboración de hormigones produce en estado fresco una disminución de la consistencia lo que se debe posiblemente al aumento de partículas finas que aumentan el poder de retención de agua. Sin embargo las mezclas presentan buena cohesión y ausencia de segregación.

Adicionar perlita a la mezcla, manteniendo constante el contenido de cemento, disminuye los tiempos de inicio y fin de fraguado e incrementa la diferencia entre ambos. Cuando se reemplaza parte del cemento por perlita, los tiempos de inicio de fraguado no se modifican sustancialmente pero sí se atrasa el fin de fraguado. Así se verifica que hay una influencia de los finos de perlita en los tiempos de fraguado que sin embargo no son significativos en la práctica de obra.

En la resistencia a compresión a edades avanzadas se produce un incremento, en contraposición con el efecto que causa en la resistencia a edades tempranas, donde la perlita no tiene influencia.

En relación al módulo de elasticidad, la incorporación de finos de perlita influye en el resultado obtenido a 28 días, disminuyendo su rigidez. Esto se muestra en la serie HP- en la cual al disminuir la cantidad de cemento, disminuye la resistencia a compresión a 28 días y lo mismo ocurre con el módulo de elasticidad.

Por lo tanto los finos de perlita pueden considerarse como una adición activa debido a su efecto puzolánico, pero como se trata de partículas muy finas sería necesario incorporar mayor cantidad de aditivo fluidificante para mantener la consistencia y así aprovechar mejor sus propiedades.

Con respecto al porcentaje de reemplazo o adición de perlita los resultados obtenidos indican que es factible su utilización y,en este caso, estaría en el orden del 5 al 10\%.Así, la posibilidad de utilizarestos residuos para la elaboración de hormigones se puede constituir en un aporte al desarrollo sustentable de la región. 


\section{Referencias}

Alonso, R. (8 de Julio de 2014). La Perlita, un vidrio natural salteño. Recuperado el 14 de Abril de 2021, de DossierWeb: https://www.dossierweb.com.ar/la-perlita-un-vidrio-naturalsalteno/

Artigas, V., Cortez, F., Burgos, L., Cardozo, H., \& Sastre, M. (2016). Diseño de pastas autocompactantes con adición de finos de perlita. VII Congreso Internacional $21^{\mathrm{a}}$ Reunión Técnica de la Asociación Argentina de Tecnología del Hormigón.

Astori, R. E., Sanguinetti, B. M., \& Bizzotto, M. (2005). La tecnología del hormigón y el desarrollo sustentable. Comunicaciones Científicas y Tecnológicas.

Bonnet, C., Carcanague, S., Hache, E., Jabberi, A., Seck, G. S., \& Simoën, M. (2019). The impact of future generation on cement demand: An assassment based on clemate scenarios.

Pal, A. (2018). Developing Low-Clinker Ternary Blends for Indian Cement Industry. J. Inst. Eng. India Ser. A.

Raggioti, B. B. (2015). Hormigones con adiciones activas: diseño, optimización y caracterización con criterio de sustentabilidad. Universidad Tecnológica Nacional Facultad Regional Córdoba.

U.S. Geological Survey. (2021). Mineral commodity summaries 2021. Reston, Virgina: U.S. Geological Survey. 